\title{
Combined Central Retinal Artery and Vein Occlusion Associated with Factor $V$ Leiden Mutation and Treated with Hyperbaric Oxygen
}

\author{
José Alberto Lemos ${ }^{a, d}$ Carla Teixeira $^{a} \quad$ Rui Carvalho ${ }^{a}$ \\ Tiago Fernandes ${ }^{\mathrm{b}, c}$ \\ Departments of ${ }^{\mathrm{a}}$ Ophthalmology, ${ }^{\mathrm{b}}$ Hyperbaric Medicine and ${ }^{\mathrm{c}}$ Anesthesiology, \\ Pedro Hispano Hospital, Matosinhos, and ${ }^{\mathrm{d}}$ Life and Health Sciences Research Institute \\ (ICVS), School of Health Sciences, University of Minho, Braga, Portugal
}

\section{Key Words}

Factor V Leiden · Hyperbaric oxygen therapy · Retinal artery occlusion · Retinal vein occlusion

\begin{abstract}
Background: Combined central retinal artery occlusion (CRAO) and central retinal vein occlusion (CRVO) is an uncommon retinal vascular disease which causes sudden visual acuity loss and is associated with poor prognosis and the development of severe complications. We report a very rare case of combined CRAO and CRVO in a patient with factor V Leiden (FVL) mutation (only 3 cases published). To our knowledge, this is the first case of combined CRAO and CRVO treated with hyperbaric oxygen therapy (HBOT). Case and Results: A 49-year-old woman presented with complaints of sudden loss of vision in her left eye (LE), with best corrected visual acuity (BCVA) of $1 / 20$. A complete ophthalmic evaluation with fundus angiography showed combined CRAO and CRVO. The patient was urgently treated with HBOT (she completed a total of 9 sessions in 7 days), with marked visual acuity and angiographic improvement (BCVA of 10/10). Forty-five days later, she developed a new LE CRVO, and BCVA decreased to $5 / 10$ and later to $<1 / 20$ because of significant macular edema. A detailed investigation showed an abnormal resistance to activated protein $C$, and a genetic study showed homozygosity for FVL mutation. The patient was submitted to 3 monthly injections of $1.25 \mathrm{mg}$ bevacizumab. After 10 months, the patient is in a stable condition with BCVA of 6/10. Conclusions: Combined CRAO and CRVO in young adults should be investigated thoroughly for embolic sources, thrombophilic disorders and local ocular conditions. This is
\end{abstract}

KARGER 125:s $\quad \begin{aligned} & \text { José Alberto Lemos, MD } \\ & \text { Rua Nossa Senhora de Fátima, } 546 \\ & \text { PT-4775-266 Viatodos, Barcelos (Portugal) } \\ & \text { E-Mail japm.lemos@gmail.com }\end{aligned}$


Lemos et al.: Combined Central Retinal Artery and Vein Occlusion Associated with Factor V Leiden Mutation and Treated with Hyperbaric Oxygen

the first case of this severe disease that was treated with HBOT, and the visual result was very good.

\section{Introduction}

Combined central retinal artery occlusion (CRAO) and central retinal vein occlusion (CRVO) is an uncommon retinal vascular disease causing sudden visual acuity loss. This is rare in young adults, and vasculitis and thromboembolic disorders are the main etiologies in these patients [1]. Factor V Leiden (FVL) mutation is a common mutation that results in a mild prohemostatic state and is a risk factor for retinal vein occlusions [2]. However, combined CRAO and CRVO in patients with FVL mutation is very rare (only 3 cases published) [1, 3]. The prognosis of combined CRAO and CRVO is very poor, and the affected eyes typically develop severe complications, such as rubeosis iridis and neovascular glaucoma [4].

We report a rare case of combined CRAO and CRVO in a patient with FVL mutation. This is the first case of combined CRAO and CRVO treated with hyperbaric oxygen therapy (HBOT).

\section{Case Presentation}

A 49-year-old female presented to the emergency department with sudden vision loss in the left eye (LE) with onset $5 \mathrm{~h}$ before. The patient also reported 2 episodes of sudden blurring of vision in her LE (10 and 8 days before) that lasted about 15 min and then fully recovered. Best corrected visual acuity (BCVA) was 10/10 in the right eye (RE) and 1/20 in the LE. An anterior segment examination revealed a left relative afferent pupillary defect. A left fundus examination revealed few intraretinal hemorrhages, mildly dilated and tortuous retinal veins, narrowed retinal arteries and a mildly cream-colored edematous appearance of the retinal posterior pole. The RE examination was normal. The past medical history included anxiety, venous peripheral insufficiency, 2 spontaneous abortions and 1 episode of deep venous thrombosis of the leg 12 years before. Her usual medication was zolpidem $10 \mathrm{mg}$ once a day.

Fluorescein angiography (FA) of the LE (fig. 1a-e) showed a delay of dye emergence in retinal arteries (19 $\mathrm{s}$ after injection) and a prolonged arteriovenous transit time with laminar flow in the retinal veins only $55 \mathrm{~s}$ after the injection. Also, venous network filling was delayed and was not completed $79 \mathrm{~s}$ after the injection (fig. 1e). No embolic material was seen. Optical coherence tomography (OCT) showed an increase in thickness and hyperreflectivity of the inner retinal layers with hyporeflectivity of the outer retinal layers (fig. 1f). This clinical picture was consistent with the diagnosis of combined CRAO and CRVO.

Initial treatment consisted of urgent HBOT, which begun $3 \mathrm{~h}$ after presentation with $100 \%$ oxygen at a pressure of 2.4 ATA for $90 \mathrm{~min}$ and acetylsalicylic acid at $150 \mathrm{mg} /$ day. Another HBOT session was done $7 \mathrm{~h}$ after the first session. There was marked improvement in vision after these HBOT sessions, with BCVA of 5/10.

The laboratory data only showed hyperlipidemia. An autoantibody screening was negative and inflammatory markers were normal. Echocardiography, neck vessel Doppler ultrasound and brain magnetic resonance imaging results were normal. The patient was sent to a hematology consult to complete thrombophilia screening. 
Lemos et al.: Combined Central Retinal Artery and Vein Occlusion Associated with Factor V Leiden Mutation and Treated with Hyperbaric Oxygen

The patient completed a total of 9 sessions of HBOT in 7 days $(2$ sessions on days 1 and 2 and then 1 daily session until stabilization of visual acuity). BCVA improved to 10/10 with significant improvement in FA (fig. 2a-c).

Forty-five days later, she developed a new LE CRVO, and BCVA decreased to 5/10 (fig. $2 \mathrm{~d}, \mathrm{e})$. At that time, she started oral anticoagulation with acenocoumarol because an underlying hypercoagulable state was suspected. After 2 weeks, the thrombophilia screening results were available and revealed an abnormal resistance to activated protein C (1.21; normal value: $>2.14$ ), and a genetic study showed homozygosity for FVL mutation. After 2 months, BCVA decreased to $<1 / 20$ because of significant macular edema (fig. 2 f), and the patient was submitted to 3 monthly injections of $1.25 \mathrm{mg}$ bevacizumab.

At present (after 10 months), the patient is in a stable condition with BCVA of 6/10; the hemorrhages were absorbed, the dilatation and tortuosity of the retinal veins had subsided (fig. 3a, b), and OCT showed no macular edema (fig. 3c). Humphrey 24-2 visual field testing showed nasal and paracentral scotoma (fig. 3d). Oral anticoagulation will become a life-long treatment in this patient in order to prevent recurrences.

\section{Discussion}

We reported a rare case of combined CRAO and CRVO in a patient with FVL mutation. The diagnostic criteria for this disease are acute unilateral visual loss with ischemic whitening of the retinal posterior pole, with or without a cherry-red spot on the macula, retinal hemorrhages, enlarged and tortuous retinal veins in 4 quadrants, delayed arterial dye filling and prolonged arteriovenous transit time on FA $[3,5]$. Our case met all these criteria and showed simultaneous signs of CRAO (mildly edematous retinal posterior pole displaying a cream-colored appearance and prolonged retinal arterial transit time in FA) and mild signs of CRVO (a few intraretinal hemorrhages, mildly dilated and tortuous retinal veins and delayed venous network filling in FA). The patient was considered to have developed incomplete CRAO with mild CRVO, followed by complete CRVO after 45 days.

Iijima and Tsumura [6] described similar cases. A possible common mechanism in those cases may be that intravenous thrombus formation is accelerated by the decreased blood flow induced by incomplete CRAO, and that the abrupt increase in retinal arterial blood flow results in increased intravenous pressure followed by augmented hemorrhages and venous dilation [6]. However, in our case the increase in retinal hemorrhages and venous dilation only occurred 45 days later, which is why we think this was a new episode of CRVO and not an exacerbation of the previous one. Nevertheless, this mechanism may explain why a slight increase in retinal hemorrhages was observed $24 \mathrm{~h}$ after initiating HBOT.

This is only the 4th reported case of combined CRVO and CRAO associated with FVL mutation $[1,3]$. Due to the high frequency of severe visual impairment in this disease, the rapid onset of severe complications and the lack of an alternative therapy, an effective emergency treatment capable of reversing the visual loss is urgently needed. HBOT has been used during the past decades to treat patients with CRAO, and $65 \%$ of all treated cases have shown improvement [7]. This is the first described case of combined CRAO and CRVO treated with HBOT. The rationale behind this treatment is that if supplemental oxygen is provided, oxygen from the choroidal circulation may diffuse in adequate quantity to the retinal inner layers to maintain retinal function and restore vision. We believe that our patient had developed combined CRAO and CRVO that was mild and that allowed significant recovery of function with the help of the supplemental oxygen provided by HBOT, which maintains retinal viability until obstructed retinal arteries recanalize. 
Lemos et al.: Combined Central Retinal Artery and Vein Occlusion Associated with Factor V Leiden Mutation and Treated with Hyperbaric Oxygen

In conclusion, combined CRVO and CRAO in young adults should be investigated thoroughly for embolic sources, thrombophilic disorders as well as local ocular conditions. Timely diagnosis and rapid intervention may help to significantly restore visual function and prevent complications. Our case was treated with HBOT, and the visual result was very good.

\section{Acknowledgements}

The authors thank to Dr. Ana Duarte and Dr. Joana Oliveira, Orthotic Technicians (Department of Ophthalmology, Pedro Hispano Hospital, Matosinhos, Portugal) for their important contribution to the acquisition of data for this work.

\section{Statement of Ethics}

The authors state that the patient gave her informed consent both to the off-label use of bevacizumab and to the publication of this case report. The authors also state that this publication was conducted in accordance with the tenets of the Declaration of Helsinki and approved by our committee on human research.

\section{Disclosure Statement}

None of the authors have any conflict of interest with the submission of this case report, and no financial support was received for this paper.

\section{References}

1 Desai S, Rai N, Kulkarni P, Natarajan S: Combined CRVO with CRAO in a patient with protein C deficiency. Retin Cases Brief Rep 2014;8:145-149.

-2 Kolar P: Risk factors for central and branch retinal vein occlusion: a meta-analysis of published clinical data. J Ophthalmol 2014;2014:724780.

-3 Schmidt D: Comorbidities in combined retinal artery and vein occlusions. Eur J Med Res 2013;18:27.

-4 Brown CG, Duker JS, Lehman R, Eagle RC Jr: Combined central retinal artery-central vein obstruction. Int Ophthalmol 1993;17:9-17.

5 Vallée JN, Paques M, Aymard A, Massin P, Santiago P-Y, Adeleine P, Gaudric A, Merland J-J: Combined central retinal arterial and venous obstruction: emergency ophthalmic arterial fibrinolysis. Radiology 2002;223:351-359.

-6 Iijima H, Tsumura T: Combined occlusion of the central retinal artery and vein. Jpn J Ophthalmol 1994;38:202-207.

7 Murphy-Lavoie H, Butler F, Hagan C: Central retinal artery occlusion treated with oxygen: a literature review and treatment algorithm. Undersea Hyperb Med 2012;39:943-953.

This case was presented in an oral communication at the 'Reunião dos Grupos Portugueses de Retina e Vítreo e de Órbita e Oculoplástica', Figueira da Foz (Portugal), October 16-17, 2015. 


\section{Case Reports in \\ Ophthalmology}

\begin{tabular}{l|l}
\hline \multicolumn{2}{l}{ Case Rep Ophthalmol 2015;6:462-468 } \\
\hline DOI: $10.1159 / 000442788$ & $\begin{array}{l}\text { C } 2015 \text { S. Karger AG, Basel } \\
\text { www.karger.com/cop }\end{array}$ \\
\hline
\end{tabular}

Lemos et al:: Combined Central Retinal Artery and Vein Occlusion Associated with Factor V Leiden Mutation and Treated with Hyperbaric Oxygen
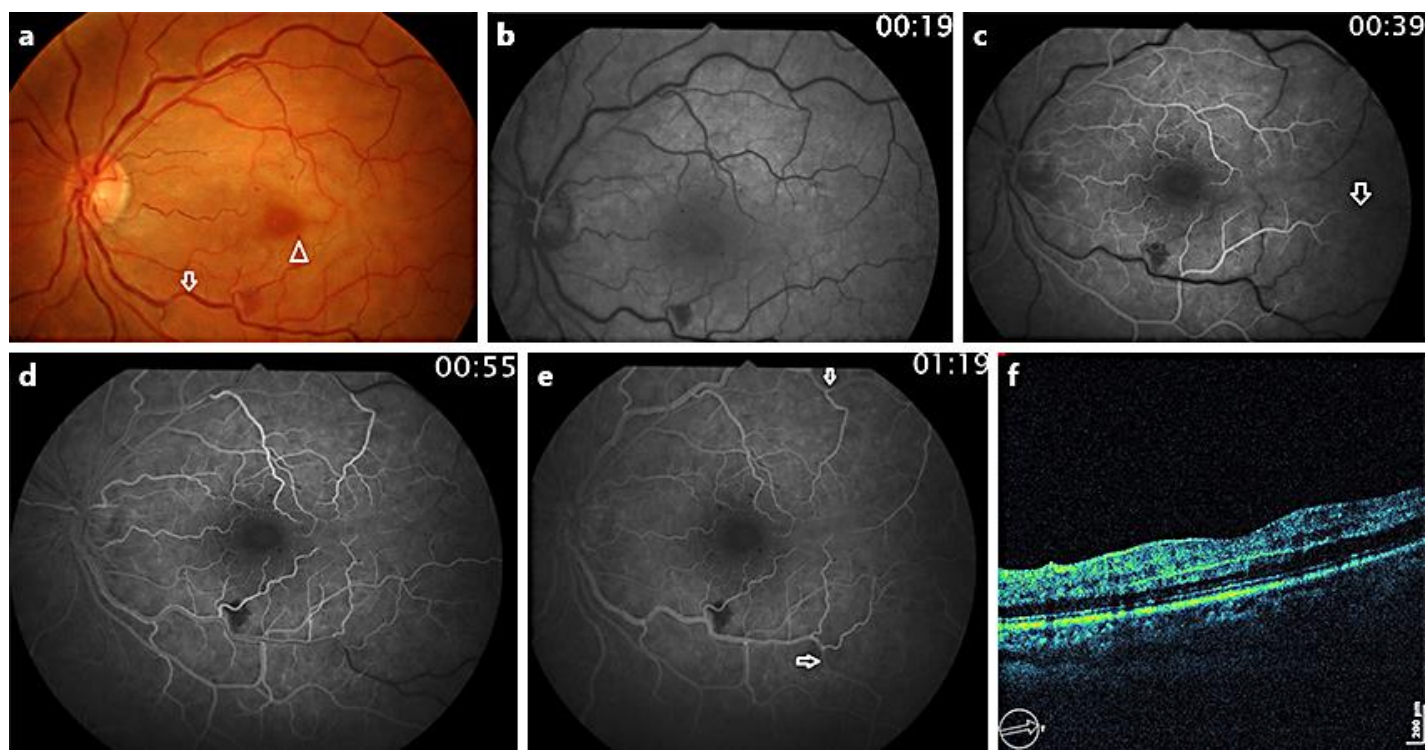

Fig. 1. Initial features. a Fundus photograph showing moderate dilatation and a tortuous venous network (arrow), few intraretinal hemorrhages (arrowheads) and ischemic whitening of the retinal posterior pole. b, c Retinal arterial phase angiograms. Retinal arterial filling is delayed and is not completed $20 \mathrm{~s}$ after emergence of the dye in the retinal arteries at the optic disk (c, arrow). This finding indicates severe impairment of the retinal arterial supply. d, e Retinal arteriovenous phase angiogram. The arteriovenous transit time, from emergence of the dye in the retinal arteries to the appearance of venous laminar flow at the optic disk, is prolonged to $36 \mathrm{~s}$ (normal: $\leq 2 \mathrm{~s}$ ). There is no capillary occlusion at the posterior pole. Venous network filling is delayed and is not completed $24 \mathrm{~s}$ (normal: $\leq 15 \mathrm{~s}$ ) after the appearance of venous laminar flow (e, arrows). f OCT image showing hyperreflectivity of the inner retinal layers and hyporeflectivity of the outer retinal layers. 
Lemos et al.: Combined Central Retinal Artery and Vein Occlusion Associated with Factor V Leiden Mutation and Treated with Hyperbaric Oxygen
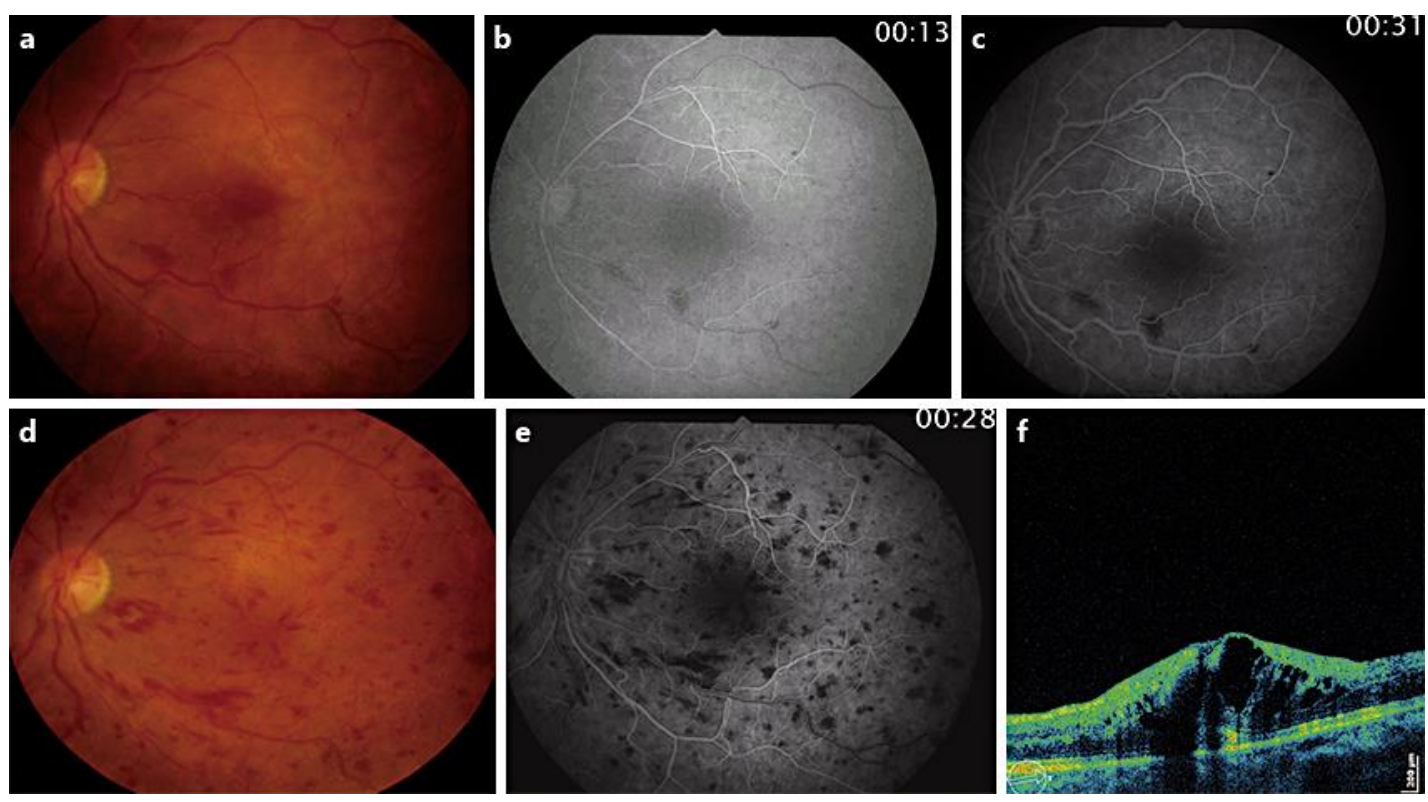

Fig. 2. a-c After HBOT. a Fundus photograph obtained $24 \mathrm{~h}$ after initiating the therapy, showing a slight increase in retinal hemorrhages. b, c Fluorescein angiograms of the fundus obtained at day 8 (after completing HBOT). b Retinal arteriovenous phase. Retinal arterial filling is beginning shortly after choroidal filling and is rapidly completed; $13 \mathrm{~s}$ after dye injection, we already observe the early appearance of venous laminar flow. The arteriovenous transit time has returned to normal at $2 \mathrm{~s}$. c Retinal venous phase. Venous network filling has greatly improved but is still slightly prolonged (18 s; normal: $<15 \mathrm{~s})$. $\mathbf{d}-\mathbf{f}$ New CRVO 45 days later. $\mathbf{d}$ Fundus photograph showing 1 peripapillary cotton-wool spot, intraretinal hemorrhages and tortuous and dilated retinal veins. e Fluorescein angiogram at $28 \mathrm{~s}$ showing an increased transit time of fluorescein flow from the arterial to the lamellar venous phase with delayed venous filling. f OCT 2 months after the new CRVO, showing significant macular edema with a central foveal thickness of $585 \mu \mathrm{m}$. 
Lemos et al.: Combined Central Retinal Artery and Vein Occlusion Associated with Factor V Leiden Mutation and Treated with Hyperbaric Oxygen
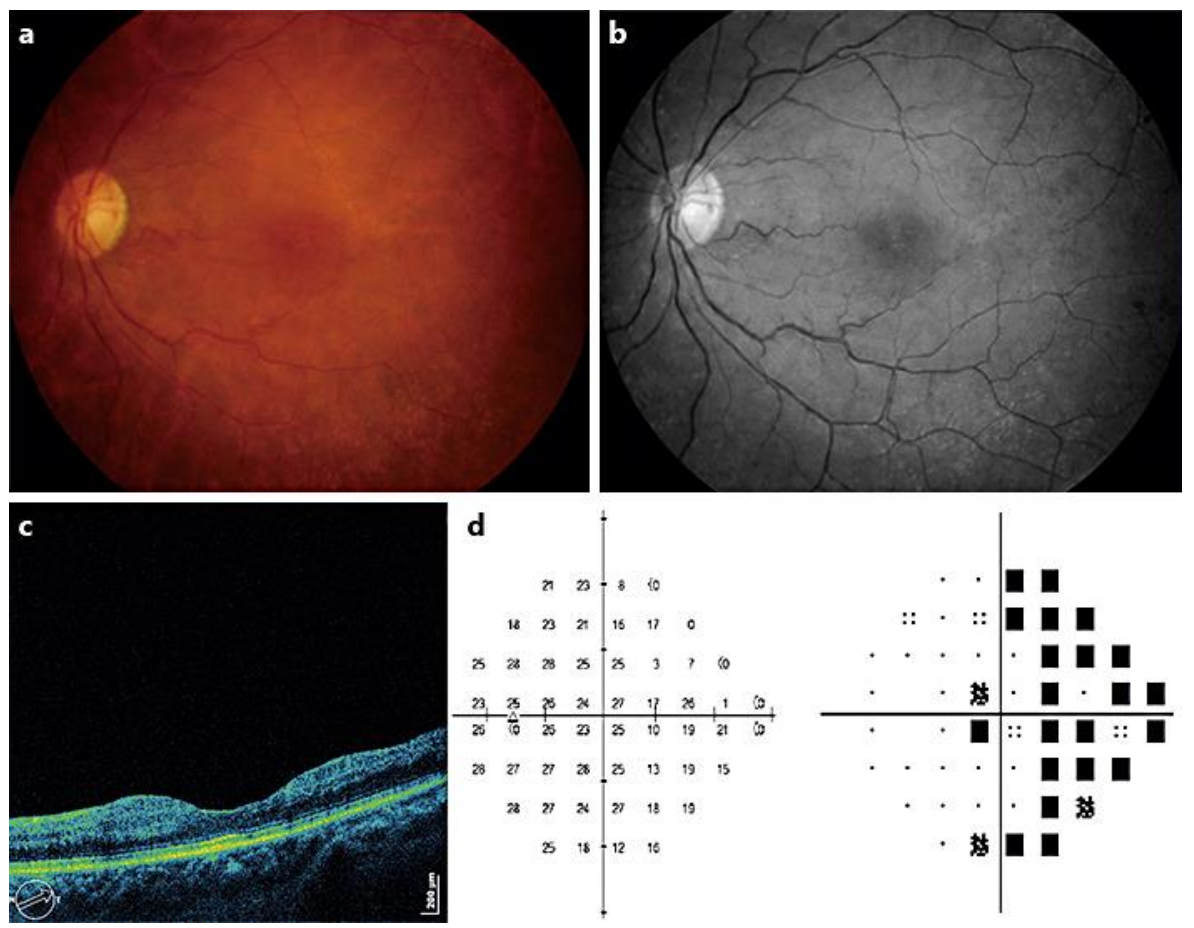

Fig. 3. After 10 months. a, b Fundus (a) and red-free (b) photographs showing mild arterial retinal narrowing without intraretinal hemorrhages or macular edema. c OCT showing absence of macular edema. d Humphrey 24-2 visual field test (SITA standard strategy) numerical sensitivity plot and pattern deviation plot showing nasal and paracentral scotoma caused by retinal ischemia. 